\title{
Modelling Light Scattering in the Context of Freeform Optical Design
}

\author{
Simon Kronberg ${ }^{1, *}$, Martijn J.H. Anthonissen ${ }^{1, * *}$ Jan H.M. ten Thije Boonkkamp ${ }^{1, * * *}$, and Wilbert L. IJzerman ${ }^{1,2, * * * *}$ \\ ${ }^{1}$ Eindhoven University of Technology (TU/e), Department of Mathematics and Computer Science, PO Box 513, \\ 5600 MB Eindhoven, The Netherlands \\ ${ }^{2}$ Signify Research, High Tech Campus 7, 5656 AE Eindhoven, The Netherlands
}

\begin{abstract}
We present an initial attempt to model scattering in freeform optical design. Scattering is modelled as a convolution of the unperturbed specular distribution and a spreading function. Deconvolution is used to recover the equivalent specular distribution, for which design procedures are well established.
\end{abstract}

\section{Introduction}

Most freeform optical design relies on geometrical optics, where light is represented by rays which travel in straight lines in homogeneous media and adhere to the law of reflection and Snell's law of refraction at optical interfaces. This approach, whilst mathematically appealing, neglects light scattering, which could negatively affect the performance of the optical element. We present an initial attempt to include these effects via a convolution of an unperturbed specular distribution and a spreading function. The design problem would thus entail prescribing a desired distribution, deconvolving it with the spreading function to get an "equivalent specular problem" and using previously developed tools in the rest of the design process.

\section{Mathematical model}

Our model is currently restricted to two-dimensional problems, but it may readily be generalised to three dimensions. We propose to model the scattering phenomena as a two-step process, whereby the incident ray first experiences a specular reflection, followed by a scattering event. The specular reflection may be described by a map $m: t \rightarrow \theta,[1]$, where $t$ and $\theta(t)$ are the directions of the incident and reflected ray, see Fig. 1. Similarly, the inverse specular mapping reads $t=m^{-1}(\theta)$.

Fix $t$, such that the energy balance in the associated specular direction $\theta=\theta(t)$ becomes

$$
\tilde{\mathcal{G}}(\theta)=\mathcal{I}\left(m^{-1}(\theta)\right) \frac{\mathrm{d} m^{-1}}{\mathrm{~d} \theta}(\theta)
$$

where $\tilde{\mathcal{G}}(\theta)$ is the specular intensity distribution as governed by the mapping $m$ and the initial source distribution $\mathcal{I}(t)$. In reaching (1), we utilised the inverse specular map

\footnotetext{
*e-mail: s.kronberg@tue.nl

**e-mail: m.j.h.anthonissen@tue.nl

***e-mail: j.h.m.tenthijeboonkkamp@tue.nl

****e-mail: wilbert.ijzerman@signify.com
}

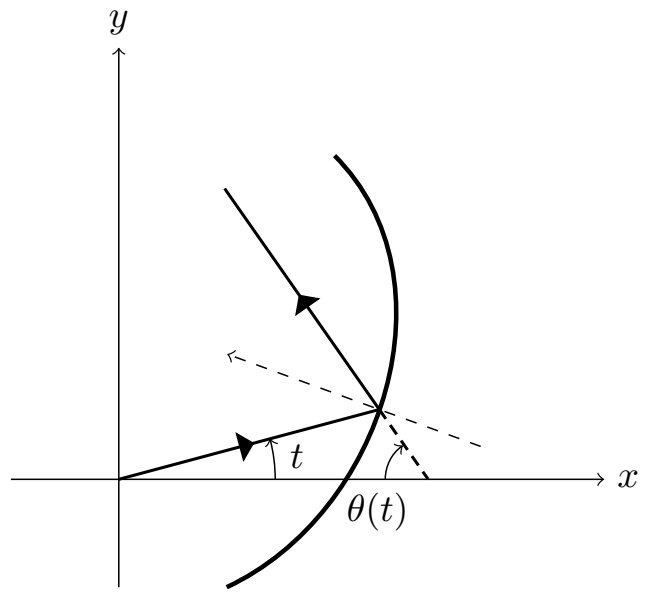

Figure 1. Illustration of the law of reflection.

and its first derivative whilst considering how a small pencil of rays centred about $t$ gets mapped by $m$.

The scattering event essentially creates a second map

$$
s_{\alpha}: \theta \rightarrow \beta, \quad \beta \equiv \theta+\alpha,
$$

where $\alpha$ is a stochastic variable representing the angular deviation from the specular direction. By again fixing $t$ for the case with scattering included, and considering the integral over the source distribution $\mathcal{I}(t)$, we get

$$
\int_{\mathcal{T}} \mathcal{I}(t) \mathrm{d} t=\int_{\mathcal{B}} \int_{\mathcal{A}} p(\alpha) \mathcal{I}\left(m^{-1}(\beta-\alpha)\right) \frac{\mathrm{d} m^{-1}}{\mathrm{~d} \theta}(\beta-\alpha) \mathrm{d} \alpha \mathrm{d} \beta,
$$

where the spreading function $p(\alpha)$ is a probability density function, and the limits of the integrals are taken to be the physically relevant directions.

Next, we state the global energy balance as

$$
\int_{\mathcal{T}} \mathcal{I}(t) \mathrm{d} t=\int_{\mathcal{B}} \mathcal{G}(\beta) \mathrm{d} \beta
$$


(a)

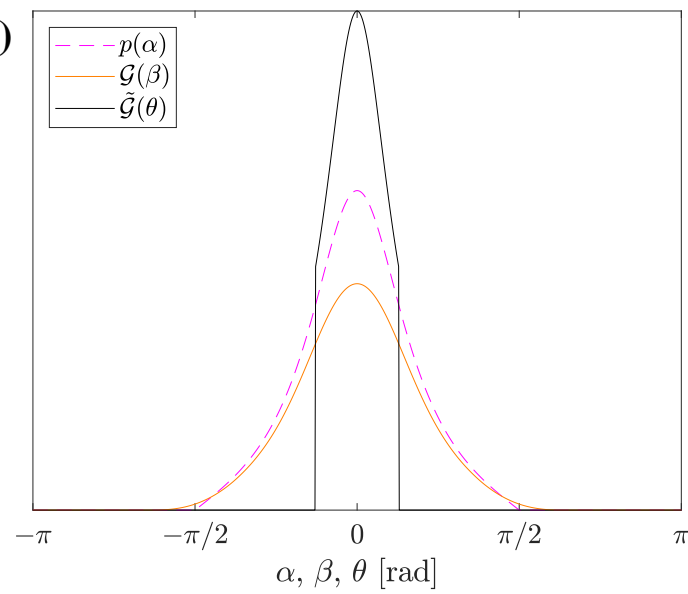

(b)

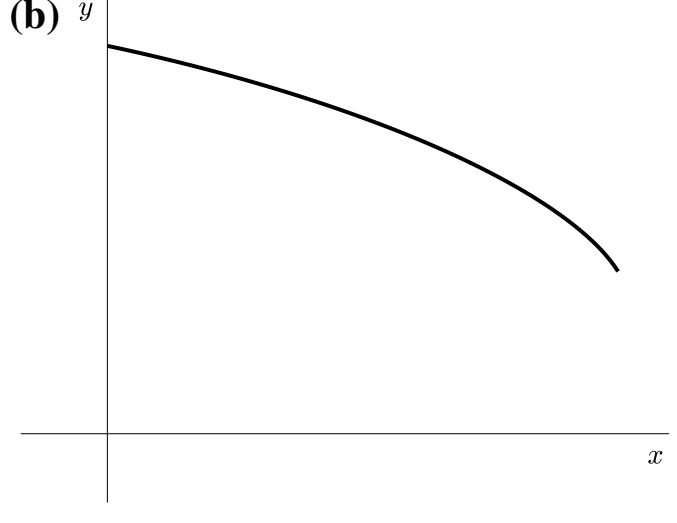

Figure 2. Two-dimensional example of reflector design using the proposed method; (a) the given intensity distribution $\mathcal{G}(\beta)$ and the spreading density function $p(\alpha)$ are deconvolved to get $\tilde{\mathcal{G}}(\theta)$; (b) the corresponding specular reflector yielding $\tilde{\mathcal{G}}(\theta)$ in the far field when $\mathcal{I}(t)$ is taken to be unity for all relevant values of $t$.

where $\mathcal{G}(\beta)$ is a prescribed intensity distribution after reflection and scattering. Comparing (2) and (3), it is clear that

$$
\begin{aligned}
\mathcal{G}(\beta) & =\int_{\mathcal{A}} p(\alpha) \mathcal{I}\left(m^{-1}(\beta-\alpha)\right) \frac{\mathrm{d} m^{-1}}{\mathrm{~d} \theta}(\beta-\alpha) \mathrm{d} \alpha \\
& =\int_{\mathcal{A}} p(\alpha) \tilde{\mathcal{G}}(\beta-\alpha) \mathrm{d} \alpha,
\end{aligned}
$$

where

$$
\tilde{\mathcal{G}}(\beta-\alpha):=\mathcal{I}\left(m^{-1}(\beta-\alpha)\right) \frac{\mathrm{d} m^{-1}}{\mathrm{~d} \theta}(\beta-\alpha)
$$

is a specular distribution without the effect of scattering. To reach this expression, recall that $\beta-\alpha=\theta$, and compare the above expression to that in (1). The two are clearly identical.

\section{Solution algorithm}

The proposed solution algorithm may be outlined as follows. Given $\mathcal{I}(t), \mathcal{G}(\beta)$ and $p(\alpha)$ :

1) Compute the deconvolution from (4) to get $\tilde{\mathcal{G}}(\theta)$.

2) Design the specular reflector with target distribution $\tilde{\mathcal{G}}(\theta)$ using contemporary tools and techniques, such a Monge-Ampère solver [2].

The critical step for the present discussion naturally becomes computing the deconvolution, where physical constraints such as positivity are crucial.

\section{Two-dimensional example}

An example of the proposed method is outlined in Fig. 2, where the equivalent specular intensity distribution $\tilde{\mathcal{G}}(\theta)$ is found by deconvolving the spreading function $p(\alpha)$ from the desired intensity distribution $\mathcal{G}(\beta)$. In this case, the deconvolution can be carried out via Matlab's \operator, but this is not generally the case as the matrix representation of $p(\alpha)$ often is nearly singular [3]. Then a simple design algorithm from Maes [1] based on conservation of energy is applied to obtain the specular reflectors in (b).

In particular, the desired intensity distribution $\mathcal{G}(\beta)$ is shown in Fig. 2 (a), together with a normalised bidirectional transmission distribution function measured from a $1.5 \mathrm{~mm}$ thick polycarbonate sample with $0.3 \%$ (weight) silicon scatterer particles acting as $p(\alpha)$. The equivalent specular distribution $\tilde{G}(\theta)=4 \pi /\left(\pi^{2}+64 \theta^{2}\right)$ has compact support $-\pi / 8 \leq \theta \leq \pi / 8$. $\mathcal{I}(t)$ is taken as unity for $\pi / 4 \leq t \leq \pi / 2$ and zero for all other $t$.

\section{References}

[1] M. Maes, Mathematical Methods for Reflector Design (CIP-gegevens Koninklijke Bibliotheek, Den Haag, 1997), ISBN 90-74445-36-5

[2] L.B. Romijn, J.H.M. ten Thije Boonkkamp, W.L. IJzerman, J. Opt. Soc. Am. A 36, 1926 (2019)

[3] P. Jansson, Deconvolution of Images and Spectra (Academic Press, 1997), ISBN 9780123802224 